\title{
Association between body mass index and muscularity in healthy older Japanese women and men
}

Hiroaki Kanehisa* and Tetsuo Fukunaga

\begin{abstract}
Background: Body mass index (BMI), expressed as the ratio of body mass to height squared $\left(\mathrm{kg} / \mathrm{m}^{2}\right)$, involves not only fat but also lean mass. The present study aimed to clarify how BMI is associated with total muscle mass (TMM) in older Japanese women and men.

Findings: Using a B-mode ultrasound apparatus, muscle thickness was measured at nine sites (forearm, upper arm anterior and posterior, thigh anterior and posterior, lower leg anterior and posterior, abdomen, and subscapular) for 346 women (BMl 16.40 to $33.11 \mathrm{~kg} / \mathrm{m}^{2}$ ) and 286 men (BMl 16.86 to $31.18 \mathrm{~kg} / \mathrm{m}^{2}$ ) aged 60.0 to $79.5 \mathrm{yrs}$. TMM was estimated using the product of the sum of the muscle thicknesses at the nine sites with height as an independent variable. For both sexes, the estimated TMM relative to height squared was significantly correlated with BMI ( $r=0.688, P<0.0001$ for women; $r=0.696, P<0.0001$ for men), but the percentage of the estimated TMM in body mass was not.
\end{abstract}

Conclusion: These results indicate that, for older Japanese women and men, BMI is a simple and convenient index for assessing total muscularity.

Keywords: Muscle thickness, Estimated muscle mass, B-mode ultrasonography

\section{Background}

Body mass index (BMI) is widely used for assessing overweight and obesity [1]. However, the association between BMI and the percentage of body fat mass (\%BF) in body mass is influenced by age, sex, and ethnicity [2-7]. Some studies have suggested that the diagnostic accuracy of BMI to detect excess body adiposity diminishes with increasing age of the person being assessed $[8,9]$. Romero-Corral et al. [9] found that the correlation between $\mathrm{BMI}$ and \%BF was lower in older than in younger subjects, but the correlation between BMI and lean mass was similar across age groups. Micozzi and Harris [10] suggested that, based on anthropometric measurements, the ratios of body mass to height ${ }^{2}$ and height ${ }^{1.5}$ in men and women, respectively, are more closely correlated with estimates of body fat in younger than in older adults, and with estimates of muscle mass in older than in younger

\footnotetext{
* Correspondence: hkane@nifs-k.ac.jp

National Institute of Fitness and Sports in Kanoya, 1 Shiromizu, Kanoya, Kagoshima 891-2393, Japan
}

adults. This suggests that, for the elderly individual, BMI may be a simple and convenient index for assessing muscularity. This assumption has already been examined using populations aged 60 and over [10-13]. IannuzziSucich et al. [11] indicated that BMI is a strong predictor of skeletal-muscle mass in older Caucasian women and men. In their study, using dual X-ray absorptiometry (DXA), BMI was shown to account for $48 \%$ and $50 \%$ of the variance in appendicular skeletal muscle mass in women and men, respectively. However, in other studies, the $r^{2}$ values between BMI and muscle mass were not so high $\left(r^{2}=0.22\right.$ to 0.40$)[10,12,13]$. The reasons for these lower $r^{2}$ values are unknown, but it might be due to the fact that the previous studies used muscle girth or area estimated from the anthropometric data of the upper arm $[10,13]$ or the upper arm and calf [12].

Gallagher et al. [4] reported that the middle-aged and older Japanese population had a higher \%BF for any given BMI than did white and African-American populations. Considering this, whether the findings of Iannuzzi-Sucich et al. [11] can be applied to the elderly Japanese 
population remains unclear. Furthermore, it seems strange that the previous studies cited above correlated the measures obtained from a limited number limbs with BMI, which is an index representing the total body. To our knowledge, no study has examined how total muscle mass is associated with BMI in elderly individuals. Thus, the present study aimed to examine the association between BMI and muscle mass in elderly Japanese individuals of both sexes. To this end, we determined muscle thicknesses at nine sites of the body using B-mode ultrasonography. The product of the sum of muscle thicknesses at these nine sites with height was shown to have a high correlation with total muscle mass (TMM) in a sample of Japanese women and men [14]. The equation with the product of the two variables as an independent variable for predicting TMM, developed in the previous study [14], has been successfully used to examine age-related muscle loss in Japanese men and women aged 20 to 95 years [15]. Using of the prediction equation, therefore, the present study estimated TMM for an older population. We hypothesized that, for the elderly population, the estimated TMM relative to height squared would be significantly correlated with BMI, but its value relative to body mass would not.

\section{Methods}

\section{Ethics approval}

This study was approved by the Ethics Committee of the Graduate School of Arts and Sciences, University of Tokyo, Japan, and was consistent with the institutional ethics requirements for human experimentation in accordance with the Declaration of Helsinki. The subjects were fully informed of the purpose and risks of the experiment, and gave their written informed consent.

\section{Subjects}

A group of 346 women and 286 men aged 60.0 to 79.5 years voluntarily participated in this study. None of the subjects was or had been an athlete. Moreover, none was using walking sticks or other walking aids, and all were functionally independent in daily life. In addition, no participant was on an extreme diet or using any major medications, such as chemotherapy, cardiac, respiratory, or antipsychotic drugs. The mean \pm standard deviation (SD) for age, height, body mass, and BMI are presented in Table 1.

\section{Muscle thickness measurements}

Muscle thickness was measured at nine sites (forearm, upper arm anterior and posterior, thigh anterior and posterior, lower leg anterior and posterior, abdomen, and subscapular) on the right side of the body, using a real time B-mode ultrasound apparatus (SSD-500, Aloka Co., Tokyo, Japan). The position of the subjects
Table 1 Descriptive data on the measured variables ${ }^{a, b}$

\begin{tabular}{|c|c|c|}
\hline Variables & Women, $\mathrm{n}=346$ & Men, $\mathrm{n}=\mathbf{2 8 6}$ \\
\hline Age, years & $69.4 \pm 4.9$ & $69.8 \pm 4.7$ \\
\hline Height, cm & $150.1 \pm 5.4$ & $163.4 \pm 6.0$ \\
\hline Body mass, kg & $53.6 \pm 7.1$ & $63.5 \pm 8$ \\
\hline $\mathrm{BMI}, \mathrm{kg} / \mathrm{m}^{2}$ & $23.77 \pm 2.77$ & $23.76 \pm 2.58$ \\
\hline \multicolumn{3}{|l|}{ Muscle thickness, mm } \\
\hline Forearm & $19.1 \pm 3.4$ & $22.6 \pm 4.2$ \\
\hline Upper arm anterior & $28.4 \pm 3.8$ & $33.3 \pm 4.6$ \\
\hline Upper arm posterior & $26.9 \pm 4.7$ & $32.8 \pm 5.1$ \\
\hline Thigh anterior & $38.1 \pm 5.6$ & $42.1 \pm 6.1$ \\
\hline Thigh posterior & $56.6 \pm 7.0$ & $61.9 \pm 7.5$ \\
\hline Lower leg anterior & $25.9 \pm 2.8$ & $28.7 \pm 3.2$ \\
\hline Lower leg posterior & $59.5 \pm 4.6$ & $66.0 \pm 5.6$ \\
\hline Subscapular & $18.2 \pm 4.3$ & $21.1 \pm 4.7$ \\
\hline Abdomen & $7.6 \pm 1.7$ & $10.4 \pm 2.1$ \\
\hline $\mathrm{SMT}^{\mathrm{c}}, \mathrm{mm}$ & $280.3 \pm 21.4$ & $319.8 \pm 27.7$ \\
\hline $\mathrm{TMM}^{\mathrm{d}}, \mathrm{kg}$ & $13.8 \pm 2.6$ & $20.8 \pm 3.7$ \\
\hline $\mathrm{TMM} / \mathrm{ht}^{2}, \mathrm{e} \mathrm{kg} / \mathrm{m}^{2}$ & $6.10 \pm 0.99$ & $7.77 \pm 1.15$ \\
\hline$\%$ TMM $^{f}$ & $25.7 \pm 3.1$ & $32.7 \pm 3.5$ \\
\hline
\end{tabular}

Values are mean \pm SD.

${ }^{b}$ All listed variables except for age and BMI were significantly greater $(P<0.0001)$ in men than in women.

'Sum of the muscle thickness values at nine sites.

${ }^{\mathrm{d}}$ Estimated total muscle mass.

'TMM relative to height squared.

${ }^{f}$ Percentage of TMM in body mass.

during the ultrasonographic measurements, the site selected for obtaining cross-sectional images, and determination of muscle thickness at each site were the same as those described in a previous study [15]. The sum of muscle thicknesses at the nine sites (SMT) was used to estimate TMM. TMM was estimated using the prediction equation developed by Sanada et al. [14]: TMM $(\mathrm{kg})=0.687 \times$ SMT $(\mathrm{cm}) \times$ body height $(\mathrm{m})-$ $15.122\left(R^{2}=0.96\right.$, SEE $\left.=1.1 \mathrm{~kg}\right)$. In addition to the absolute value, the estimated TMM relative to height squared $\left(\mathrm{TMM} / \mathrm{ht} \mathrm{t}^{2}, \mathrm{~kg} / \mathrm{m}^{2}\right)$ and the percentage of the estimated TMM in body mass (\%TMM) were calculated, and used to examine how muscularity and the relative distribution of muscle mass within body mass are associated with BMI.

\section{Statistics}

Descriptive values are presented as mean \pm SD. An unpaired Student's $t$-test was used to test the differences between men and women in the measured variables. A simple linear regression analysis was used to calculate the coefficient of correlation between BMI and TMM/ht ${ }^{2}$ or \%TMM. The probability level for statistical significance was set at $P<0.05$. 


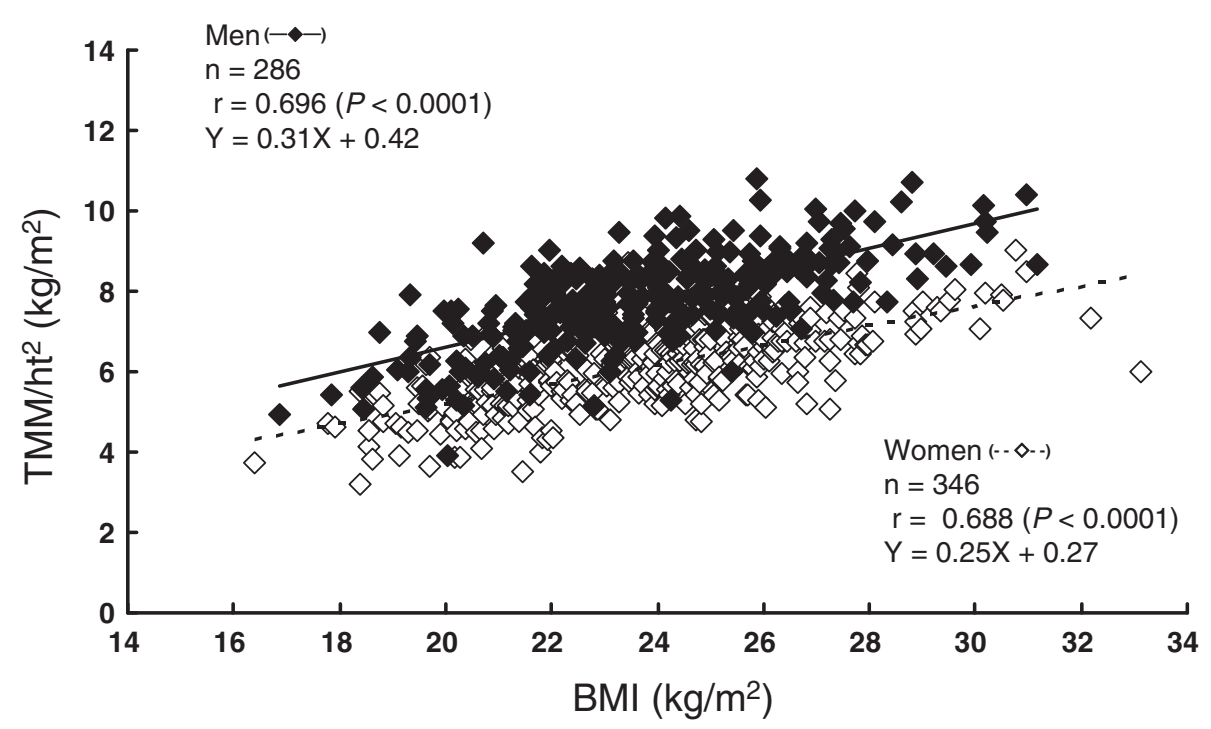

Figure 1 Relationship between $\mathrm{BMI}$ and $\mathrm{TMM} / \mathrm{ht}^{2}$.

\section{Results}

All measured variables except for age and BMI were significantly greater in men than in women (Table 1 ). $\mathrm{TMM} / \mathrm{ht}^{2}$ was significantly correlated with BMI in both women $(r=0.688, P<0.0001)$ and men $(r=0.696$, $P<0.0001$ ) (Figure 1). However, \%TMM was not significantly associated with BMI in either sex: $r=-0.024$ $(P>0.05)$ for women and $r=-0.029(P>0.05)$ for men (Figure 2).

\section{Discussion}

The current results support our hypothesis regarding the association between BMI and muscle mass, and indicate that, for the elderly person, BMI is an index that assesses total muscularity rather than the relative distribution of muscle mass within the total body. Based on the $r^{2}$ values, BMI accounted for $47 \%$ and $48 \%$ of the variance in $\mathrm{TMM} / \mathrm{ht}^{2}$ in women and men, respectively. These values are higher than those reported in previous studies using anthropometric approaches for predicting muscle size $[10,12,13]$, and are comparable those in the study of Iannuzzi-Sucich et al. [11] who used DXA to determine appendicular skeletal muscle mass. In the present study, we estimated the total muscle mass by using a prediction equation developed by Sanada et al. [14]. Based on the report of Sanada et al. [14], the accuracy of the ultrasonography prediction model for estimating TMM is greater than that of anthropometric

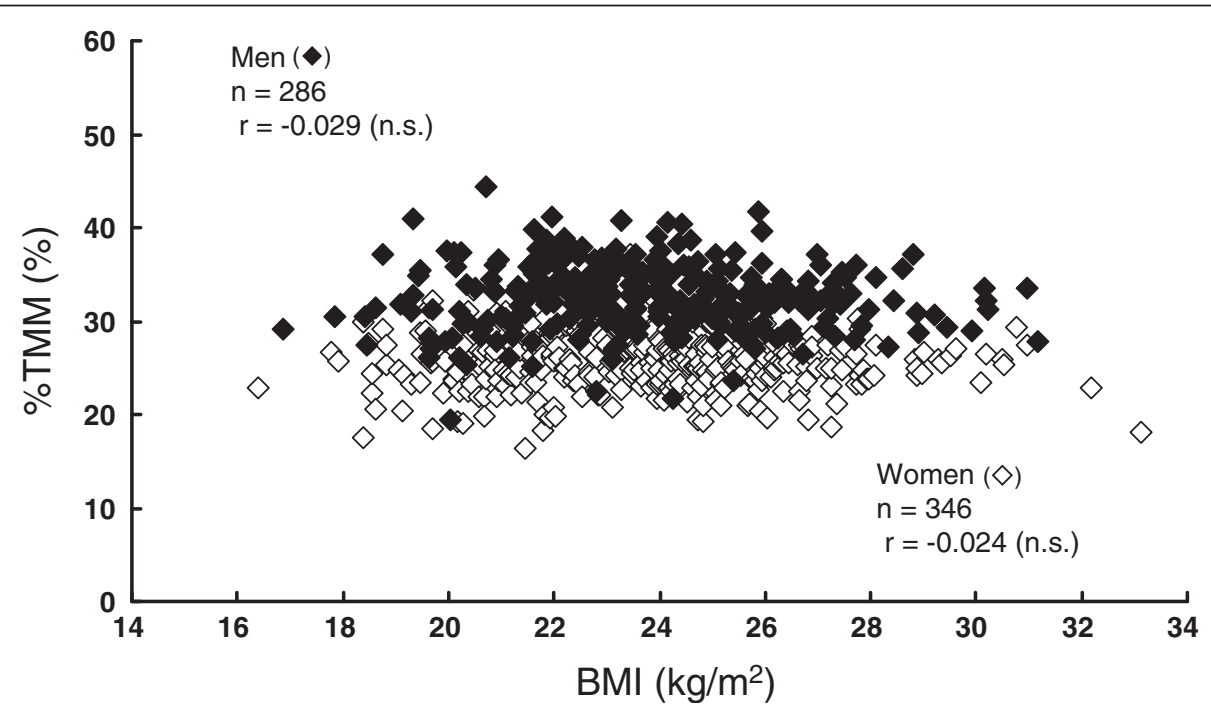

Figure 2 Relationship between BMI and \%TMM. 
and bioelectrical impedance prediction models, and is similar to that of DXA prediction models and wholebody $40 \mathrm{~K}$ counting. This may explain the aforementioned difference in the observed $r^{2}$ between the current study and previous reports using anthropometric model. However, it should be noted that the number of subjects in our study categorized as underweight $\left(\mathrm{BMI}<18.50 \mathrm{~kg} / \mathrm{m}^{2}\right)$ or obese $\left(30.00 \mathrm{~kg} / \mathrm{m}^{2}\right.$ or higher) [1] was small $(23 ; 3.6 \%)$. The correlation coefficient of the associations between BMI and TMM $/ \mathrm{ht}^{2}$ was found to be lower for subjects with BMI greater than 25 (women: $r=0.468, P<0.001$, men: $r=0.448, P<0.0001)$ than for subjects with BMI less than 25 (women: $r=0.660, P<0.0001$, men: $r=0.630$, $P<0.0001)$. This implies a limitation on the use of BMI as an index for assessing muscularity in elderly individuals with relation to the magnitude of BMI. Further studies examining individuals categorized as either underweight or obese are needed to clarify this finding.

\section{Abbreviations}

\%BF: Percentage of body fat mass in body mass; BMI: Body mass index; TMM: Total muscle mass; SMT: Sum of muscle thicknesses at the nine sites; TMM $/ \mathrm{ht}^{2}$ : Estimated TMM relative to height squared; \%TMM: Percentage of the estimated TMM in body mass.

\section{Competing interests}

The authors declare that they have no competing interests.

\section{Authors' contributions}

HK participated in study design, helped acquire funding from the Ministry of Education, Culture, Sports, Science and Technology, helped coordinate research activities, performed statistical analysis, and drafted the manuscript. TF participated in study design and coordination, and drafted the manuscript. Both authors read and approved the final manuscript.

\section{Acknowledgement}

This research was supported in part by financial aid from the Ministry of Education, Culture, Sports, Science and Technology of Japan (no. 1248007).

Received: 14 September 2012 Accepted: 15 February 2013

Published: 4 March 2013

\section{References}

1. WHO expert consultation: Appropriate body-mass index for Asian populations and its implication for policy and intervention strategies. Lancet 2004, 363:157-163.

2. Deurenberg P, Weststrate JA, Seidell JC: Body mass index as a measure of body fatness: age- and sex-specific prediction formulas. Br J Nutr 1991, 65:105-114.

3. Gallagher D, Visser M, Sepulveda D, Pierson RN, Harris T, Heymsfield SB: How useful is body mass index for comparison of body fatness across age, sex, and ethnic groups? Am J Epidemiol 1996, 143:228-239.

4. Gallagher D, Heymsfield SB, Heo M, Jebb SA, Murgatroyd PR, Sakamoto Y: Healthy percentage body fat ranges: an approch for developing guidelines on body mass index. Am J Clin Nutr 2000, 72:694-701.

5. Jackson AS, Stanforth PR, Gagnon J, Rankinen T, Leon AS, Rao DC, Skinner JS, Bouchard C, Wilmore JH: The effect of sex, age and race on estimating percentage body fat from body mass index: The Heritage Family Study. Int J Obes Relat Disord 2002, 26:789-796.

6. Meeuwsen S, Horgan GW, Elia M: The relationship between BMI and percent body fat, measured by bioelectrical impedance, in a large adult sample is curvilinear and influenced by age and sex. Clin Nutr 2010, 29:560-566.

7. Rush E, Freitas I, Plank LD: Body size, body composition and fat distribution: comparative analysis of European, Maori, Pacific Island, and Asian Indian adults. Br J Nurt 2009, 102:632-641.
8. Baumgartner RN, Heymsfield SB, Roche AF: Human body composition and the epidemiology of chronic disease. Obes Res 1995, 3:73-95.

9. Romero-Corral A, Somers VK, Sierra-Johnson J, Thomas RJ, Bailey KR, Collazo-Clavell NL, Allison TG, Korinek J, Baysis JA, Lopez-Jimenez FL: Accuracy of body mass index to diagnose obesity in the US adult population. Int J Obes (Lond) 2008, 32:959-966.

10. Micozzi MS, Harris TM: Age variations in the relation of body mass indices to estimates of body fat and muscle mass. Am J Phys Anthropol 1990, 81:375-379.

11. lannuzzi-Sucich M, Prestwood KM, Kenny AM: Prevalence of sarcopenia and predictors of skeletal muscle mass in healthy, older men and women. J Gerontol Med Sci 2002, 57A:M772-M777.

12. Ross WD, Crawford SM, Ward KR, Bailey DA, Mirwald RM: Relationship of the body mass index with skinfolds, girths, and bone breadths in Canadian men and women aged 20-70 years. Am J Phys Anthropol 1988, 77:169-173

13. Wannamethee SG, Shaper AG, Lennon L, Whincup PH: Decreased muscle mass and increased central adiposity are independently related to mortality in older men. Am J Clin Nutr 2007, 86:1339-1346.

14. Sanada K, Kearns CF, Midorikawa T, Abe T: Prediction and varidation of total and regional skeletal muscle mass by ultrasound in Japanese adults. Eur J Appl Physiol 2006, 96:24-31

15. Abe T, Sakamaki M, Yasuda T, Bemben MG, Kondo M, Kawakami Y, Fukunaga T: Age-related, site-specific muscle loss in 1507 Japanese men and women aged 20 to 95 years. J Sports Sci Med 2011, 10:145-150.

doi:10.1186/1880-6805-32-4

Cite this article as: Kanehisa and Fukunaga: Association between body mass index and muscularity in healthy older Japanese women and men. Journal of Physiological Anthropology 2013 32:4.

\section{Submit your next manuscript to BioMed Central and take full advantage of:}

- Convenient online submission

- Thorough peer review

- No space constraints or color figure charges

- Immediate publication on acceptance

- Inclusion in PubMed, CAS, Scopus and Google Scholar

- Research which is freely available for redistribution 